\title{
MORTALIDAD POR CÁNCER EN LA MUJ ER CHILENA. ANÁLISIS COMPARATIVO ENTRE LOS AÑOS 1997 Y 2003
}

\author{
Enrique Donoso S. ${ }^{1}$, Mauricio Cuello F. ${ }^{1}$ \\ ${ }^{1}$ Departamento de Obstetricia y Ginecología, Facultad de Medicina, Pontificia Universidad Católica de Chile.
}

\begin{abstract}
RESUMEN
Antecedentes: El cáncer es la segunda causa de muerte en los países desarrollados y presenta un continuo descenso para la mayoría de ellos, independiente del sexo o raza. Objetivos: Comparar entre los años 1997 y 2003 la mortalidad por cáncer en la mujer chilena y establecer las localizaciones más frecuentemente asociadas a mortalidad. Material y Método: Las tasas de mortalidad se calcularon con el número de muertes por cáncer y la población estimada de mujeres obtenidas de los anuarios de Estadísticas Vitales de 1997 y 2003. Resultados: En el período hubo aumento significativo de la tasa general de mortalidad por cáncer en la mujer. En ambos años los 5 sitios más frecuentes de muerte por cáncer fueron: mama, estómago, vesícula biliar, broncopulmonar y cérvico uterino; solo la mortalidad por cáncer de mama aumentó significativamente, mientras que la por cáncer cérvico uterino presentó reducción significativa, los otros 3 no presentaron cambios significativos. Hubo aumento significativo de las muertes por cáncer de colon, páncreas, ovario, linfoma no Hodgkin, mieloma, encéfalo, vejiga urinaria y leucemia linfoide. Conclusiones: Con excepción del cáncer cérvico uterino, hubo un aumento significativo de la mortalidad oncológica en la mujer chilena. Esta tendencia obliga a revisar la forma de registro de eventos y los programas ya existentes, como también establecer nuevas estrategias que permitan mejorar la prevención, acceso y calidad de tratamiento del cáncer a fin de revertir esta situación. Se espera que la introducción de nuevas políticas de salud como las incorporadas en la Ley 19.996 tenga el impacto deseado.
\end{abstract}

\section{PALABRAS CLAVES: Mortalidad por cáncer, tendencia, salud pública, Chile}

\section{SUMMARY}

Background: Cancer is the second cause of death in developed countries and shows a continuous reduction in rate independently of sex and race. Objectives: To compare the cancer mortality rate observed among Chilean women and establishing the main localizations causing death in that group in 1997 and 2003. Material and Methods: The cancer mortality rate was calculated using the total number of cancer deaths and the estimated women population obtained from the vital statistical reports of 1997 and 2003. Results: Between 1997 and 2003, it was a significant increase in overall cancer mortality rate among women. In both years, the five more frequent causes of cancer death were: breast, stomach, gallbladder, lung, and cervical cancer; among them, breast cancer increased and cervical cancer decreased, both significantly; the others did not experience significant rate changes. There were significant increases in other less frequent cancers such as: colon, pancreas, ovarian, non-Hodgkin lymphoma, myeloma, brain, bladder, and lymphatic leukemia. Conclusions: Overall, there has been an increase in oncologycal mortality rate in Chilean women. With the exception of cervical cancer, the majority of the more frequent cancers have increased their mortality rate. This increasing mortality trend enforces to review the quality of the data registration, the existing health programs, and designing new strategies which will lead us to improve prevention, access, and quality of treatment, and finally to decrease cancer mortality. New strategies such that incorporated in Law 19,996 are called to change current outcomes.

KEY WORDS: Cancer mortality, trends, public health, Chile 


\section{INTRODUCCIÓN}

En Chile, el cáncer es la segunda causa de muerte después de las enfermedades cardiovasculares (1); mientras la tasa bruta de mortalidad general descendió desde 6,0/1000 en 1990 a 5,3/ 1000 habitantes en 2003 (2), la tasa de mortalidad general por cáncer aumentó de 108,1/100.000 en 1990 a 122,8/100.000 habitantes en 2002 (3). Países desarrollados de la Región, como EEUU y Canadá, presentan un descenso de la mortalidad general por cáncer $(4,5)$. En EEUU, el cáncer también es la segunda causa de muerte con una tasa de mortalidad de 193,8/100.000 habitantes en 2002 (4), y descendió $11 \%$ entre 1990 y 2000 (6); en ese período, la reducción de la mortalidad fue mayor en hombres que en mujeres y en ellas la reducción estuvo dada por un número menor de muertes por cáncer mamario y colon, responsables del $86 \%$ de la reducción en esa década (7).

Los objetivos de este estudio fue comparar la mortalidad general por cáncer en la mujer chilena entre los años 1997 y 2003, establecer los sitios más frecuentes de mortalidad y la variación de las tasas entre los dos años estudiados.

\section{MATERIAL Y MÉTODO}

Se compara la mortalidad por cáncer en la mujer chilena entre el año 1997 y 2003. Chile en 1997 adoptó la 10 ${ }^{\text {a }}$ Clasificación Internacional de Enfermedades y Causas de Defunción, motivo por lo que este estudio se inicia en ese año ya que los cambios de codificación y de los contenidos no permiten una comparación con años anteriores. El número de muertes por cáncer en mujeres y la población femenina estimada se obtuvo de los anuarios de Estadísticas Vitales correspondientes a los años en estudio y publicados por el Instituto Nacional de Estadísticas de Chile $(2,8)$. Se analizan solo los cánceres que presentaron 100 o más casos de muertes en 2003. Las tasas de mortalidad para los diferentes tipos de cánceres fueron calculadas y expresadas por 100.000 mujeres. La comparación de proporciones se efectuó según Chi cuadrado $\left(\chi^{2}\right)$ con corrección de Yates, con un nivel de significación estadística de $p<0,05$. La variación entre las tasas de mortalidad se presenta como porcentaje de cambio.

\section{RESULTADOS}

En 1997 y 2003 la población estimada de mujeres fue 7.386 .165 y 7.961 .513 , respectivamente. En 1997 el total de muertes por cáncer en mujeres fue de 8.321 casos (112,7/100.000 mujeres) y 9.580 en $2003(120,3 / 100.000$ mujeres), diferencia significativa $\left(\chi^{2}=19,2 ; p=0,00001\right)$ (Tabla I). Hubo un aumento de $6,7 \%$ de la tasa global de mortalidad entre los años estudiados, equivalente a $1,1 \%$ anual.

Los primeros 5 sitios de cánceres asociados a muerte en mujeres en 2003 fueron mama, estómago, vesícula biliar, broncopulmonar y cérvico uterino, correspondiente al $47,4 \%$ del total de fallecimientos; mientras que en 1997 fueron estómago, mama, vesícula biliar, cérvico uterino y broncopulmonar, equivalentes al $49,4 \%$ del total de fallecimientos (Tabla II). El año 2003 comparado con 1997, presenta una reducción significativa de las muertes por cáncer cérvico uterino; mientras que hubo aumento significativo de las muertes por cáncer de mama, colon, páncreas, ovario, de sitios no especificados, linfoma no Hodgkin, mieloma, secundario de los órganos digestivos y respiratorios, encéfalo, vejiga urinaria y leucemia linfoide; los otros cánceres no presentaron cambios significativos (Tablas II y III).

\section{DISCUSIÓN}

Este estudio analiza la mortalidad por cáncer en mujeres que en 2003 presentaron más de 100 muertes, comparado con 1997. Pudimos observar que en este período de 6 años hubo un aumento de la tasa global de mortalidad por cáncer en la mujer de 6,7\%, equivalente a $1,1 \%$ anual. Esta tendencia es contraria a lo presentado en los últi-

Tabla I

COMPARACIÓN DE LA MORTALIDAD GLOBAL (MUERTES/100.000 MUJERES) POR CÁNCER EN LA MUJER CHILENA, 1997 y 2003

\begin{tabular}{lccc}
\hline Año & Población estimada & Muertes $^{*}$ & Mortalidad \\
\hline 1997 & 7.386 .165 & 8.321 & 112,7 \\
2003 & 7.961 .513 & 9.580 & 120,3 \\
\hline
\end{tabular}

${ }^{*} \chi 2=19,2 ; p=0,00001$. 
Tabla II

NÚMERO DE MUERTES POR CÁNCER EN MUJERES CHILENAS EN 1997 Y 2003, CON MÁS DE 100 FALLECIMIENTOS EN 2003

\begin{tabular}{lrr}
\hline Sitios & 1997 & $n$ \\
& 885 & $n$ \\
\hline Mama & 1.026 & 1.070 \\
Estómago & 877 & 1.055 \\
Vesícula biliar & 589 & 1.035 \\
Bronquios y pulmón & 733 & 702 \\
Cérvico uterino & 444 & 677 \\
Colon & 338 & 554 \\
Páncreas & 345 & 492 \\
Hígado y de las vías biliares intrahepáticas & 254 & 337 \\
Ovario & 235 & 327 \\
De sitios no especificados & 268 & 306 \\
Esófago & 148 & 292 \\
Linfoma no Hodgkin de otro tipo y del no especificado & 115 & 244 \\
Mieloma múltiple y tumores de células plasmáticas & 110 & 180 \\
Recto & 132 & 142 \\
Riñón, excepto de la pelvis renal & 105 & 136 \\
Leucemia mieloide & 79 & 127 \\
Secundario de los órganos digestivos y respiratorios & 72 & 119 \\
Encéfalo & 715 \\
Vejiga urinaria & 72 & 114 \\
Leucemia linfoide & 59 & 103 \\
\hline Fuen Insto & & \\
\hline
\end{tabular}

Fuente: Instituto Nacional de Estadísticas.

Tabla III

ANÁLISIS COMPARATIVO DE LA VARIACIÓN DE LA TASA DE MORTALIDAD POR CÁNCER EN MUJERES CHILENAS, 1997 Y 2003

\begin{tabular}{|c|c|c|c|c|}
\hline Sitios & $\begin{array}{c}1997 \\
\text { tasa }\end{array}$ & $\begin{array}{r}2003 \\
\text { tasa }\end{array}$ & $\begin{array}{l}\text { Variación } \\
\quad \pm \%\end{array}$ & $\begin{array}{l}\text { Valor } \\
p\end{array}$ \\
\hline Mama & 12,0 & 13,4 & $+11,7$ & 0,01 \\
\hline Estómago & 13,9 & 13,3 & $-4,3$ & NS \\
\hline Vesícula biliar & 11,9 & 13,0 & $+9,2$ & NS \\
\hline Bronquios y pulmón & 8,0 & 8,8 & $+10,0$ & NS \\
\hline Cérvico uterino & 9,9 & 8,5 & $-14,1$ & 0,004 \\
\hline Colon & 6,0 & 6,9 & $+15,0$ & 0,02 \\
\hline Páncreas & 4,6 & 6,2 & $+34,8$ & 0,000 \\
\hline Hígado y de las vías biliares intrahepáticas & 4,7 & 4,2 & $-10,6$ & NS \\
\hline Ovario & 3,4 & 4,1 & $+20,6$ & 0,03 \\
\hline De sitios no especificados & 3,2 & 3,8 & $+18,8$ & 0,03 \\
\hline Esófago & 3,6 & 3,7 & $+2,8$ & NS \\
\hline Linfoma no Hodgkin de otro tipo y del no especificado & 2,0 & 3,1 & $+55,0$ & 0,000 \\
\hline Mieloma múltiple y tumores de células plasmáticas & 1,6 & 2,3 & $+43,8$ & 0,002 \\
\hline Recto & 1,5 & 1,8 & $+20,0$ & NS \\
\hline Riñón, excepto de la pelvis renal & 1,8 & 1,7 & $-5,5$ & NS \\
\hline Leucemia mieloide & 1,4 & 1,6 & $+14,3$ & NS \\
\hline Secundario de los órganos digestivos y respiratorios & 1,1 & 1,5 & $+36,4$ & 0,02 \\
\hline Encéfalo & 1,0 & 1,4 & $+40,0$ & 0,01 \\
\hline Vejiga urinaria & 1,0 & 1,4 & $+40,0$ & 0,01 \\
\hline Leucemia linfoide & 0,8 & 1,3 & $+62,5$ & 0,003 \\
\hline
\end{tabular}

Tasa: muertes x 100.000 mujeres. NS: diferencia no significativa. 
mos años por los países desarrollados de la Región (4-7). Sin embargo, el último reporte realizado en los EEUU, aunque confirma una tendencia sostenida al descenso de la mortalidad global por cáncer, también muestra que cuando se subdivide por sexo, existe un incremento leve de la mortalidad por cáncer en mujeres $(9,10)$.

Las defunciones por cáncer en Chile entre 1990 y 2002 han aumentado independiente del sexo de los fallecidos; en hombres la tasa de mortalidad general por cáncer aumentó de 107,2 a $127,2 / 100.000$ y en mujeres de 109,0 a 118,6/ 100.000 (3). En este estudio observamos que los 5 sitios más frecuentes asociados a muerte se mantuvieron en los años analizados y que la mortalidad en mujeres por cáncer de mama, vesícula biliar y estómago lideran los primeros tres lugares y de estos solo las muertes por cáncer de mama aumentaron significativamente. Es notable que del total de cánceres, solamente las muertes por cáncer cérvico uterino disminuyeran significativamente, con una reducción de la mortalidad de $14,1 \%$. Mientras que las muertes por cáncer de mama, colon, páncreas, ovario, sitios no especificados, linfoma no Hodgkin, mieloma, encéfalo, vejiga urinaria y leucemia linfoide aumentaron significativamente, con un rango entre 15 y $62,5 \%$; en el resto de los cánceres los cambios no fueron significativos. Probablemente el menor incremento en la tasa de mortalidad general por cáncer en mujeres se deba al mejor control del cáncer cérvico uterino.

El reciente reporte de EEUU, realizado con los 15 cánceres más frecuentes, abarcando desde 1975 al 2002, muestra que para la última década, la mortalidad disminuyó en 9 de los 15 cánceres, incluyendo varios de los que se mantienen sin cambios o que han aumentado en nuestro país; entre ellos: cáncer de mama, colon y recto, linfoma no Hodgkin, leucemia, estómago, cerebro, mieloma, cuello uterino y vejiga (9). En EEUU sólo el cáncer de pulmón aumentó su mortalidad entre mujeres; así, en el período 1993-2002, se registró un descenso de la mortalidad global por cáncer de $1,1 \%$ con leve aumento entre las mujeres $(9,10)$. Similar tendencia en la mortalidad general por cáncer se observó en Canadá (5). Interesante resulta ver como tres de los cinco cánceres más frecuentes en la mujer chilena muestran una tendencia opuesta en los países más desarrollados de la Región. Al revisar lo que ocurre en los países de la Unión Europea, entre 1990-2000, la mortalidad global por cáncer en mujeres ha mostrado un sostenido descenso, especialmente cán- cer mamario (11\%), gástrico (31\%), colorrectal $(16 \%)$ y cérvico uterino $(20 \%)$, mientras que páncreas y ovario no han modificado su tasa de mortalidad y la de cáncer pulmonar aumentó importantemente (17\%) (11).

Surge así la pregunta ¿por qué existen estas diferencias? Las explicaciones pueden ser diversas. Los resultados de este estudio sugieren, dentro de las causales, que en Chile los programas ya existentes destinados al control del cáncer no han sido eficientes, con excepción del Programa Nacional de Cáncer Cérvico Uterino (12-14). Al analizar las 5 causas más frecuentes de muerte por cáncer en Chile observamos que:

a) Para cáncer mamario, Chile cuenta con el Programa Nacional de Cáncer de Mama. El cáncer de mama en 2003 fue la primera causa de muerte en mujeres y entre los años estudiados hubo un aumento significativo del número de fallecimientos, con un aumento de la tasa de mortalidad de $11,7 \%$. Este programa iniciado en 1995, protocoliza la pesquisa de los tumores palpables y la derivación de los casos sospechosos o con historia familiar de cáncer mamario a las Unidades de Patología Mamaria, así el $81,8 \%$ de los ingresos fue por tumor palpable y $41,4 \%$ estaba en etapa III-IV (15); solo el $14 \%$ de los cánceres se diagnosticó mediante mamografía y permitió un aumento del diagnóstico de etapas I-II de $53 \%$ en 1995 a 67,6\% en 2000 (16). En contraste, en EEUU la mortalidad por cáncer de mama disminuyó $2,1 \%$ anual en el período $1990-1997$ y $4,1 \%$ anual desde 1995 (17), con una tasa de mortalidad de 14,6/100.000 mujeres en 2002 (4), mientras que en la Unión Europea la mortalidad por cáncer de mama desciende a partir de 1989 alcanzando una tasa de mortalidad de 18,9/100.000 mujeres en 2000 (11), descensos atribuibles al diagnóstico precoz (tamizaje poblacional mediante mamografía a partir de los 40 años) y un mejor tratamiento basado en guías de práctica clínica, incorporación oportuna de nuevas tecnologías, educación continua de especialistas, fomento a protocolos de investigación, etc.

b) El cáncer gástrico es la segunda causa de muerte por cáncer en mujeres chilenas y no presentó cambios significativos del número de muertes entre los años estudiados. En Chile, la pesquisa de cáncer gástrico en etapas precoces reveló que la prevalencia de cánceres incipientes era similar en la población explorada que en los pacientes sintomáticos (18) y que la mayoría llegaba en etapas avanzadas, de bajo rendimiento terapéutico (19). En EEUU, el cáncer gástrico en mu- 
jeres entre 1990-1997, disminuyó significativamente $2,6 \%$ anual (17). Para esta entidad no existe herramienta de tamizaje y en nuestro país además no existe un programa nacional de tratamiento. Ante la ausencia de herramientas de tamizaje efectivo, la diferencia con países desarrollados ha de estar en un mejor tratamiento.

c) El cáncer de vesícula biliar y de vías biliares extrahepáticas es la tercera causa de muerte por cáncer en mujeres chilenas y presentó un aumento no significativo de las muertes. Este cáncer se asocia a la elevada incidencia de litiasis biliar en la mujer chilena, y la estrategia actual para su control es la prevención secundaria mediante colecistectomía, especialmente en el grupo etario de 45 a 49 años y al desarrollo de líneas de investigación que permitan establecer una efectiva prevención primaria (20).

d) El cáncer broncopulmonar es la cuarta causa de muerte por cáncer en mujeres chilenas con un aumento no significativo de las muertes entre los años estudiados. Este cáncer está fuertemente asociado al hábito tabáquico; en Chile el estudio nacional efectuado por la Comisión Nacional de Control de Estupefacientes en 2000, informó una prevalencia anual de $48,7 \%$ (hombres $52,7 \%$, mujeres $45,4 \%$ ), resultados muy semejantes a los presentados por funcionarios de la salud (hombres $43,0 \%$, mujeres $39,6 \%)(21,22)$. En EEUU es la primera causa de muerte por cáncer en hombres y mujeres para todos los grupos raciales con excepción de las hispánicas; en hombres presentó un continuo descenso desde 1990, mientras que en mujeres continúa su ascenso junto con el aumento de la incidencia (17). La reducción del consumo de tabaco permitirá disminuir la incidencia y la mortalidad por cáncer broncopulmonar (23). A diferencia de países desarrollados, aún hay falta de conciencia nacional para reducir el hábito tabáquico. Tanto a nivel educacional como legislativo, las herramientas útiles en desincentivar el consumo permanecen lejos de ser efectivamente aplicadas.

e) El cáncer cérvico uterino es la quinta causa de muerte por cáncer en mujeres chilenas y fue el único que presentó una disminución significativa de la mortalidad entre 1997 y 2003. Esta reducción es debida fundamentalmente al aumento de la cobertura del Papanicolaou en mujeres entre 25 y 64 años $(12,13)$, que permitió aumentar el diagnóstico en etapas precoces y así reducir la tasa de mortalidad a 8,5/100.000 mujeres en 2003, pero lejos aún de EEUU que en 2002 presentó una mortalidad de 1,4/100.000 mujeres (4) y de la Unión Europea con 5,4/100.000 mujeres en 2000
(11). Esas diferencias no sólo se explican por la cobertura insuficiente del Papanicolaou, sino también por diferencias en la calidad de tratamiento. Aun existen en nuestro país limitaciones para el acceso oportuno a tratamiento, particularmente a la radioquimioterapia en regiones.

Es claro que la implementación efectiva de programas de salud para cada entidad es requisito indispensable para reducir la mortalidad. Ello queda refrendado con los resultados recientes del Programa Nacional de Cáncer Cérvico Uterino. Contrasta con lo observado hasta ahora con otros programas como el de cáncer de mama. Sin embargo, resulta superficial el atribuir que el aumento sostenido de la mortalidad por cáncer entre mujeres se deba sólo a la falta de eficacia de los programas existentes. Cabe recordar por una parte, que para ver los efectos posterior a la implementación de un programa deben transcurrir al menos 10 años desde su implementación y por ende el análisis sea un tanto prematuro.

El aumento de la mortalidad por cáncer en la mujer chilena se explica también por otras variables, dentro de ellas: a) el envejecimiento poblacional, que entre 1997 y 2003, donde la población femenina de 40 o más años, la más susceptible para las primeras 5 causas de mortalidad por cáncer, aumentó de 2.310 .850 en 1997 (31,3\% de la población de mujeres) a 2.769.429 en 2003 (34,8\% de la población de mujeres) $(2,8)$; b) además de los cambios alimentarios poblacionales (24) y c) al aumento de otros hábitos relacionados a cáncer $(21,22)$. El envejecimiento de la población hace que la incidencia de cáncer aumente globalmente, lo que es más significativo en mujeres, donde para 2005 se estima una esperanza de vida cercana a los ochenta años. En cuanto a los hábitos, tanto el tabaquismo como el consumo de alcohol han aumentado en la mujer, ambos factores vinculados directa o indirectamente a un mayor riesgo de desarrollar cáncer. Esto ha sido demostrado en EEUU para el tabaquismo, donde los cambios en el consumo por parte de la mujer han correlacionado directamente con la mayor ocurrencia de cáncer de pulmón, fenómeno que se confirma en el último reporte donde se observan las variaciones y se analizan las tendencias a largo plazo (9).

Para implementar un programa efectivo en reducir la mortalidad es necesario que además de un diagnóstico oportuno, se acceda a un tratamiento de calidad. Dicho tratamiento debe ser basado en la evidencia. De la experiencia americana se deduce que existe un importante retraso 
en que esa información llegue al nivel de la comunidad (10). Por ello, además de desarrollar guías clínicas de manejo es importante mejorar la comunicación al interior de las redes de salud para que estas sean conocidas en forma oportuna por todos los actores. Importa mejorar el registro de los eventos, generar un registro electrónico, desarrollar la informática médica, uniformar el lenguaje, y estar alertas a los avances en investigación básica y clínica que puedan modificar la norma vigente de cuidado.

Un registro actualizado y preciso es crucial para que un programa cuente con estimados confiables sobre los cuales se base la introducción de modificaciones o nuevas estrategias. Además de registrar la mortalidad por cáncer es necesario monitorizar otras variables, dentro de ellas la incidencia de la enfermedad. Lamentablemente en Chile aun no existen registros adecuados sobre incidencia de la enfermedad; si existen reportes sobre egresos hospitalarios. Sólo recientemente, desde fines de los noventa, ha comenzado a realizarse un registro de los casos nuevos en algunas regiones del país (provincia de Valdivia, II y VIII región) (3). Los estimados nacionales para la incidencia de cáncer oscilan entre 220 a 240 por 100.000 habitantes y alrededor de 36.500 casos nuevos por año. Si se desconoce los casos nuevos, una pieza importante de la información no se toma en cuenta en el análisis. El aumento en la mortalidad podría explicarse no por ineficacia del programa, sino por un aumento del número de casos que sobrepasa al sistema.

El impacto de las políticas de salud debe evaluarse según los objetivos que ellas persiguen. Los esfuerzos gubernamentales han estado en mejorar el acceso a la salud de la población definido por la OMS como el bienestar integral de la persona. Dentro de esos conceptos se enmarca la Reforma de Salud puesta en marcha en julio de 2005; Ias Garantías Explícitas en Salud, establecidas en la Ley 19.996 (25), dentro de las 25 prioridades sanitarias identificadas, garantiza la atención del cáncer cérvico uterino, del cáncer mamario, de los linfomas en mayores de 15 años, del cáncer en menores de 15 años, del cáncer de testículo en mayores de 15 años y del alivio del dolor por cáncer avanzado y cuidados paliativos; esas acciones debieran reflejarse en el descenso de la mortalidad de esos cánceres y en la calidad de vida de los pacientes, especialmente en mujeres, como también en el cumplimiento de los objetivos sanitarios 2000-2010 de reducir la tasa de mortalidad estandarizada por edad en $40 \%$ para cáncer cérvico uterino y en $25 \%$ para cáncer mamario y vesicular (26).

\section{BIBLIOGRAFÍA}

1. Medina E, Kaempffer AM. Mortalidad por cáncer en Chile: consideraciones epidemiológicas. Rev Med Chile 2001; 129: 1195-202.

2. Instituto Nacional de Estadísticas. Estadísticas Vitales. Chile, 2003.

3. Ministerio de Salud. Programa nacional de cáncer. Hallado: www.minsal.cl

4. Kochanek KD, Smith BL. Deaths: preliminary data for 2002. National Vital Statistics Reports; vol. 52 , no. 13. Hyattsville, Maryland: National Center for Health Statistics. 2004.

5. National Cancer Institute of Canada. Canadian cancer statistics, 2004. Hallado en: www.cancer.ca

6. Chu KC, Baker SG, Tarone RE. A method for identifying abrupt changes in U.S. cancer mortality trends. Cancer 1999; 86: 157-69.

7. Andersen LD, Remington $P$, Trentham-Dietz $A$, Reeves M. Assessing a decade of progress in cancer control. The Oncologist 2002; 7: 200-4.

8. Instituto Nacional Estadísticas. Demografía. Chile, 1997.

9. Edwards BK, Brown ML, Wingo PA, Howe HL, Ward E, Ries LAG, Schrag D, et al. Annual report to the nation on the status of cancer, 1975-2002, featuring population-based trends in cancer treatment. J Natl Cancer Inst 2005; 97: 1407-27.

10. Zielinski SL. US cancer mortality continues decline but incidence rises slightly for women. J Natl Cancer Inst 2005; 97(19):1393-93.

11. Levi F, Luchini F, Negri E, La Vecchia C. Trends in mortality from major cancers in the European Union, including acceding countries, in 2004. Cancer 2004; 101: 2843-50.

12. Suárez E, Prieto M, Rojas I, Fernández B, Prado R, Olfos P. Programa nacional de cáncer cervicouterino. Rev Chil Obstet Ginecol 2001; 66: 480-91.

13. Capurro I, Rojo JA, Pino T, Vásquez C, Garay J, Venegas M. Programa de detección y control de cáncer de cuello uterino en Servicio de Salud Araucanía Sur. Rev ChIL Obstet Ginecol 2002; 67: 114-20.

14. Guzmán S, Salas $P$, Puente R, Hott H, Israel E, Guzmán R. Pesquisa y control del cáncer cérvicouterino en el Servicio de Salud de Valdivia. Rev Med Chile 2005; 133: 685-92.

15. Peralta O, Jorquera A, Rencoret C, Del Castillo C Solé J, Campodónico I. Cáncer de mama. Resultados del programa de pesquisa y tratamiento del Servicio de Salud Central. Rev Chil Obstet Ginecol 1995; 60: 417-27.

16. Peralta O. Cáncer de mama en Chile. Datos epidemiológicos. Rev ChIL OBstet Ginecol 2002; 67: 439 45.

17. Ries LAG, Wingo PA, Miller DS, Howe HL, Weir HK, 
Rosenberg HM, Vernon SW, et al. The annual report to the nation on the status of cancer, 1973-1997, with special section on colorectal cancer. Cancer 2000; 88: 2398-424.

18. Llorens P. Resultados del examen masivo en el diagnóstico de cáncer gástrico. En: Csendes A, Strauszer T, eds. Cáncer gástrico. Santiago: Andrés Bello, 1984.

19. Csendes A, Braghetto I, Smok G, Nava O, Medina E. Estudio cooperativo en cáncer gástrico incipiente e intermedio: aspectos clínicos, diagnósticos y terapéuticos. Rev Med Chile 1992; 120(4): 397-406.

20. Montoya-Aguilar C. La litiasis biliar y el cáncer de vesícula y vías biliares en Chile: dos estrategias. Cuad Méd Soc Chile 2004; 44:162-75.

21. CONACE. Cuarto estudio nacional de consumo en drogas en Chile, informe ejecutivo. 2000.
22. Bello S, Soto M, Michalland S, Salinas J. Encuesta nacional de tabaquismo en funcionarios de salud. Rev Med Chile 2004; 132: 223-32.

23. Tyczynski JE, Bray F, Parkin M. Lung cancer in Europe in 2000: epidemiology, prevention and early detection. Lancet Oncol 2003; 4: 45-55.

24. Albala C, Vio F, Kain J, Uauy R. Nutrition transition in Latin America: the case of Chile. Nut Rev 2001; 59:170-6.

25. República de Chile. Ley 19.996. Garantías explícitas en salud del régimen de garantías en salud. Hallado en: www.minsal.cl

26. Ministerio de Salud, Gobierno de Chile. Objetivos sanitarios para la década 2000-2010. Hallado en: http://epi.minsal.cl/epidemiologia. 